flow in a cardiac system may serve both to increase mixing and to maintain organized spatial patterns.

Hassan Aref is in the Department of Theoretical and Applied Mechanics, University of Illinois at

Urbana-Champaign, 104 South Wright Street, Urbana, Illinois 61801, USA.

e-mail:h-aref@uiuc.edu
1. Rothstein, D., Henry, E. \& Gollub, J. P. Nature 401, 770-772 (1999).

2. Aref, H. J. Fluid Mech. 143, 1-21 (1984).

3. Arnold, V. I. C. R. Acad. Sci. A 261, 17-20 (1965).

4. Hénon, M. C. R. Acad. Sci. A 262, 312-314 (1966)

5. Eckart, C. J. Mar. Res. 7, 265-275 (1948).

6. Ottino, J. M. The Kinematics of Mixing (Cambridge Univ. Press, 1989).

7. Aref, H. \& El Naschie, M. S. (eds) Chaos Applied to Fluid Mixing (Pergamon, Oxford, 1995).

Space biology

\title{
Life without gravity
}

Richard J. Wassersug

$\mathrm{N}$ ow is a quiet period in space biology, perhaps as quiet as before autumn 1957, when Laika the dog — and the discipline - left the launch pad. The primary platforms for biological research in microgravity are retired (Mir and NASA's modular Spacelab), on indefinite hold (Russia's unmanned BION satellites), or still being made (the International Space Station). Even NASA's space shuttles are grounded for maintenance. So this is a good point at which to take stock of where space life science has been and where it might go. As part of this process, NASA's Center for Advanced Studies in the Space Life Sciences sponsored an unusual workshop last month* on how microgravity affects biological systems - unusual in that it focused on microgravity's effects above the cellular level, in contrast to most contemporary biology, which is more molecular and reductionist in scope.

Research in space biology is often rationalized on the grounds that it may lead to medical advances for debilitating malaises of old age (such as osteoporosis and muscle wasting), or that it may improve the health and welfare of astronauts on long missions. Those arguments, although potentially valid, were hardly heard at the workshop. Rather, the common motivating force seemed to be a genuine desire to understand the role that gravity has played in the evolution of life on our planet, using microgravity as an investigative tool.

The workshop was unusual not only in its focus, but also in the diversity of organisms and organ systems discussed. Attendees heard about molluscs, insects, fish, amphibians, rats, monkeys, humans and even wheat ${ }^{1-4}$. The word 'integrative' in the workshop's title refers to the direct and indirect effects of microgravity and other environmental variables on multiple organ systems and their development. A nice example of this is seen in medaka. This fish was the first

* Microgravity's Effects on Biological Systems and Behavior: An Integrative Approach. NASA Center for Advanced Studies in the Space Life Sciences at the Marine Biological Laboratory, Woods Hole, Massachusetts, 2-4 September 1999. (and still the only) vertebrate to mate and lay eggs that developed into free-living offspring in microgravity ${ }^{5}$. Under these conditions, some strains of medaka continuously somersault as they swim. The problem is not in their vestibular system, as one might suppose, but in their visual system (K. Ijiri, Univ. Tokyo) - strains with poor vision are more likely to loop.

Another example is wheat. This is the first species to be raised to seed, from seed that was itself raised in microgravity (G. Bingham, Utah State Univ.). That simple goal of getting a large terrestrial plant to complete two generations in space - had eluded space botanists for decades. We now know that water requires special management in microgravity. A granular matrix around roots, even a seemingly well-hydrated one, can have non-wet voids that hinder water uptake. And capillarity, unopposed by gravity, can produce films of fluid that block gas diffusion and limit oxygen uptake by roots. Similarly, in microgravity germinating seeds easily asphyxiate.

There is evidence from recent missions such as Neurolab that, during certain critical periods, gravity is necessary for normal development of the vestibular-motor reflexes in both frogs (E. Horn, Univ. Ulm) and rats (A. Ronca, NASA Ames Research Center, California). Developmental biologists at the workshop spoke for longer-term studies into this gravity dependence. Likewise, the comparative physiologists identified a multitude of questions that could be answered given longer-term missions. For instance, does the rate of bone demineralization in astronauts carry an increased risk of kidney-stone formation (C. Wade, NASA Ames Research Center, California)? Does the well-documented wasting of postural muscle in microgravity feed back on the nervous system such that reconditioning is retarded on re-entering 'normal' gravity (R. Roy, Univ. California, Los Angeles)? And does the unexpectedly high deposition of small (less than $1 \mu \mathrm{m}$ ) aerosol particles in the lungs under microgravity increase the risk of chest infections in astronauts (J. West, Univ. California, San Diego)?
Perhaps the most impressive demonstration of what can be accomplished came from neurobehavioural studies. On the $1998 \mathrm{Neu}-$ rolab mission, four rats had multi-electrode recording arrays chronically implanted next to their hippocampal 'place cells' (neurons that encode a cognitive map of the environment), to see which cells fired as the animals negotiated a three-dimensional track (J. Knierim, Univ. Texas, Houston). Such studies could reveal how information on gravity (and other accelerations) is processed by the nervous system, from the peripheral receptor to deep within the brain. They could also help to explain the visual illusions experienced by some astronauts when they arrive in space - such as the impression that the world is upside down - and how the nervous system accommodates to microgravity as these illusions disappear later in the mission.

Despite a general consensus about what can realistically be accomplished in integrative space biology, there were subtle differences of opinion about what should fly on the International Space Station. For example, developmental biologists felt that research in the near term must use only a limited set of model organisms. In contrast, several physiologists and behaviourists felt that many species are needed, covering a large size range, so that scaling effects can be dissected out. Both are correct. For most studies of early embryogenesis, a rat is a rat. But for studies in physiology or behaviour, the differences between laboratory and wild strains of the same species may be enormous.

Although integrative space biology is barely 40 years old, the workshop showed that it has a pedigree of performance, as well as realistic goals. It was also agreed that the International Space Station should be an outstanding laboratory for integrative biology, despite its limitations for other scientific disciplines. To understand how microgravity affects biological systems we need the time, work space, equipment and on-site attention available only in a high-quality, manned space station with good internal environmental controls - just what the International Space Station is supposed to be.

Richard J. Wassersug is in the Department of Anatomy and Neurobiology, Dalhousie University, Halifax, Nova Scotia, Canada B3H 4 H7.

\section{e-mail:tadpole@is.dal.ca}

1. Souza, K., Hogan, R. \& Ballard, R. (eds) Life into Space: Space Life Sciences Experiments, NASA Ames Research Center 1965-1990 (Government Printing Office, Moffett Field, California, 1995).

2. Moore, D., Bie, P. \& Oser, H. (eds) Biological and Medical Research in Space: An Overview of Life Sciences Research in Microgravity (Springer, Germany, 1996).

3. Stark, R. E. Ethology in Space, a Unique Opportunity for Behavioural Science (ESA, Noordwijk, The Netherlands, 1993). 4. Nicogossian, A. E., Huntoon, C. L. \& Pool, S. L. (eds) Space Physiology and Medicine (Lea \& Febiger, Philadelphia, 1994). 5. Ijiri, I. The First Vertebrate Mating in Space: A Fish Story (RICUT, Tokyo, 1995). 